\title{
High-speed Railway External Power Supply Reliability Evaluation of Bayesian Network
}

\author{
Zechuan Liang, Minwu Chen, Guoxu Shang, Baoyu Lv \\ School of Electrical Engineering, Southwest Jiaotong University, Chengdu, China \\ Email: liangzechuan@foxmail.com
}

Received April, 2013

\begin{abstract}
Reliability evaluation is important in high speed railway external power supply design, based on probability reasoning bayesian network applied in high-speed railway external power supply reliability evaluation, establish the minimum cut and the minimum path of bayesian network model, quantitative calculation external power supply system in each element posterior probability, and the example analysis verified the feasibility and correctness of the above method. Using bayesian network bidirection reasoning technology, quantitative calculation the posterior probability of each element in external power supply system, realized the identification of weak link in external power supply. The research methods and the results of the study can be used in the scheme optimization design of high speed railway external power supply.
\end{abstract}

Keywords: Bayesian Networks; The Minimal Path; The Minimal Cut; External Power Source; Reliability Evaluation

\section{Introduction}

With the large-scale construction of electrified railway in our country, especially, the high-speed railway opening, reliability problem increasingly cause the attention of people [1]. As the first level of power load, electrified railway traction power supply system needs to provide stable and reliable power system of the external power supply. Due to the high speed rail (including passenger dedicated line) operating speed is high, so the external power supply in the event of failure, may result in traction power supply system of power supply interruption, directly affect high-speed railway security, reliable and efficient operation. So on the outside of the high speed railway power supply scheme is essential in the design of power supply reliability assessment, the quantitative calculation of the external power supply reliability, accurate identification of external power supply of the weak links, optimizing the design of the external power supply scheme, has important theoretical significance and application value.

Through based on probabilistic inference of bayesian network used in high-speed railway external power supply reliability evaluation, aiming at traction substation power supply without interruption, establishing bayesian networks model, writing the reliability calculation program, quantitative evaluation caused by external power failure probability of traction power substation. Bidirectional reasoning technology based on bayesian network, identification the weak links of high speed railway ex- ternal power supply to optimize the design of high speed railway external power supply scheme.

\section{Summary of Bayesian Network}

In recent years, the method of bayesian network has been successful used in many fields. bayesian network in literature [2] is applied to fault diagnosis of power grid, bayesian network in literature [3] is applied to reliability evaluation of distribution network, bayesian network in literature [4] is used in power system reliability assessment. After years of development and perfection, bayesian networks has become an effective evaluation of reliability, general method.

Bayesian networks also called the reliability network is a kind of probability network, it is based on probabilistic graphical network [4]. A bayesian network is a directed acyclic graph, it is represented by variables of nodes and connecting the nodes of directed arcs.

Bayesian use nodes to represent variables and directed arcs between nodes represent the relationship between variables, through the graphical expression of uncertain knowledge, through annotations of the conditional probability distribution can be expressed local conditions dependency in the model[5].

Because bayesian network has bidirectional reasoning technology, so it can be calculated after the occurrence of certain events $\mathrm{T}, \mathrm{X}$ occurs the a posteriori probability of the event, specially, 1 represents the event occurs, specific as follows: 
Posterior probability from the Angle of the fault diagnosis reflects the importance of the components in the system size, especially suitable for weak links in recognition system, fault diagnosis and inspection and repair planning[6].

\section{Bayesian Network Model and Algorithm}

Based on the traditional shortest path set method or the minimum cut set method when evaluating the reliability of the system, finally will involve the minimization algorithm of path sets and minimum cut sets processing, toquantitative calculation of system reliability finally. But when the system is very complex, the element number is larger, the minimization algorithm processing will be complicated. Because bayesian network itself contains the conditional independence between the node variable precision, so based on shortest path set method or minimum cut set method of bayesian networks need not minimum cut set or sets the path minimization algorithm processing[7].

Based on shortest path set method or minimum cut set method of bayesian network model using the path set method or minimum cut set method of bayesian networks is established. Specific steps are as follows[8]:

1) Finding out the path sets and minimum cut sets of the system;

2) Setting the components in the path set and minimum cut as initial model element node;

3) Setting all the node path sets and minimum cut as subsystem node;

4) Using conditional probability tables to describe the relationship of the subsystem node and the initial element nodes.

Logical relationship in here is mainly refers to "or" and "and", conversions with the bayesian network are shown in Figure 1[9]:
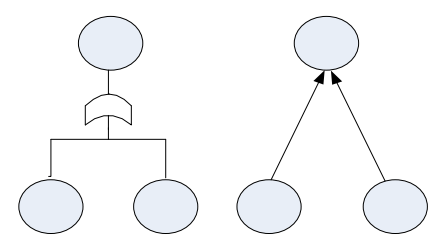

(a) Or gate
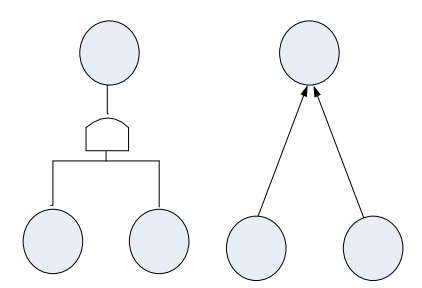

(b) And gate

Figure 1. Logic relation and Bayesian network transformation.
After convert the path sets and minimum cut sets to bayesian networks, reliability indices can be reasoning calculationcan. The algorithm about precise reasoning of bayesian networks is mainly based on Poly Tree Propagation, Bucket-Elimination, Clique Tree Propagation. Bayesian networks are precise reasoning algorithm is mainly based on Poly real Tree Propagation method, the method based on combinatorial optimization problems, and based on Clique Tree Propagation method. The method of Poly Tree Propagation is only applicable to simply connected, Bucket - Elimination and Clique Tree Propagation fit with both simply connected also with multiply connected, but when there are multiple asked node in the system, often using a Clique Tree Propagation method.

\section{Example Analysis}

\subsection{High Speed Railway External Power Supply Reliability Index}

Using the reliability evaluation model of international general IEEE RBTS - (6) system as an example, putting forward high-speed railway external power supply scheme which is based on shortest path set method of bayesian network and bayesian network based on minimal cut set method to quantitative evaluation the scheme of power supply reliability of the traction substation, respectively.

RBTS (6 nodes) system are shown in Figure 2:

Figure 2: a power on bus 1, 2, one bus in the power plant has four generating units, they are G11、G12、G13、 G14, and its capacity are P11, P12, P13 and P14, P11 = $\mathrm{P} 12$ = $40 \mathrm{MW}, \mathrm{P} 13$ = $20 \mathrm{MW}, \mathrm{P} 14=10 \mathrm{MW}$; Bus 2

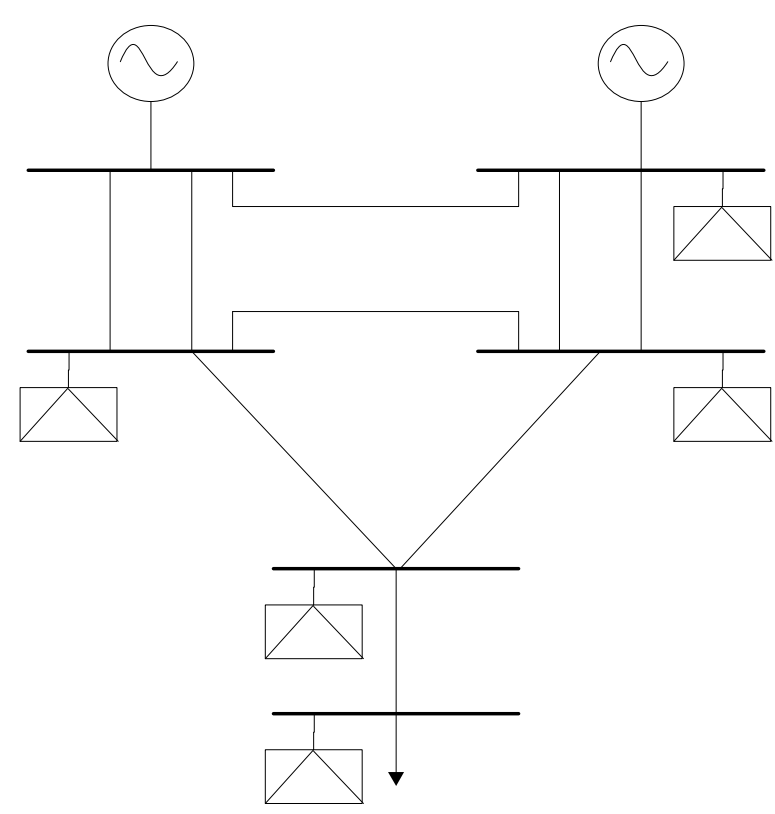

Figure 2. RBTS (6 node) system. 
there are 7 station in power plant, namely, G21, G22, G23, G24, G25, G26 and G27, its capacity is P21 , P22, $\mathrm{P} 23, \mathrm{P} 24, \mathrm{P} 25, \mathrm{P} 26$ and $\mathrm{P} 27$, the $\mathrm{P} 21=40 \mathrm{MW}, \mathrm{P} 22=$ $\mathrm{P} 23=\mathrm{P} 24=\mathrm{P} 25,=20 \mathrm{MW} 、 \mathrm{P} 26=\mathrm{P} 27=5 \mathrm{MW}$. The reliability of components of the system parameters are shown in Tables 1-2[10]. Assumes that the bus bars in Figures 2-4 respectively with the traction substation TPSS2 - TPSS6, these traction substation to supply one high speed railway.

\subsection{Bayesian Network Based on Shortest Path Set Method}

Based on high-speed rail as shown in Figure 2 external power supply network, can respectively calculate the traction substation power supply of the path set, here in traction substation TPSS2 on bus 2 as an example, using Boolean determinant method, can get the shortest path sets of TPSS2: G1L3, G1L1L2L4 G1L1L4L7, G1L2L4L6, G1L1L2L5L8 G1L1L5L7L8, G2, G1L4L6L7, G1L2L5L6L8 G1L5L6L7L8. According to the shortest path of TPSS2 set can make corresponding bayesian networks, shown as in Figure 3.

Figure 3: L1, L2, L3,... , L9 respectively represent L1 - L9 in the system of RBTS (6 nodes); A1, A1, A2,... A5, representing the path G1L3, G1L1L2L4, G1L1L4L7, G1L2L4L6 G1L4L6L7, G1L1L2L5L8; the relationship $\mathrm{A} 1, \mathrm{~A} 2, \ldots, \mathrm{A} 5$ and $\mathrm{T}$ is "or". In combination with the conditional probability tables of $\mathrm{A} 1, \mathrm{~A} 2, \ldots, \mathrm{A} 5, \mathrm{~T}$ and the failure rate of components, and connecting with the development of reliability assessment based on bayesian networks we can calculate $\mathrm{P}(\mathrm{T})_{2}$ :

$$
\mathrm{P}(\mathrm{T}) 2=6.6348 \times 10^{-6}
$$

Among them, $\mathrm{P}(\mathrm{T})_{2}$ is the outage probability of the traction substation on bus 2. Similarly, we can calculate the probability of power traction substation outage on the bus 3 to 6 bus on as follows:

$$
\begin{aligned}
& \mathrm{P}(\mathrm{T})_{3}=6.6348 \times 10^{-6} \\
& \mathrm{P}(\mathrm{T})_{4}=6.6348 \times 10^{-6} \\
& \mathrm{P}(\mathrm{T})_{5}=6.7206 \times 10^{-6} \\
& \mathrm{P}(\mathrm{T})_{6}=2.9671 \times 10^{-4}
\end{aligned}
$$

which we can see, the probability of power outage on the bus $6(\mathrm{P}(\mathrm{T}) 6)$ is greater than the rest of the few relatively traction substation, because the bus 6 use single wire(L9) accessing, so the failure rate is relatively large.

Table 1. IEEE-RBTS (6 node) system line data

\begin{tabular}{cccc}
\hline Line & \multicolumn{2}{c}{ Bus from to } & $\begin{array}{c}\text { Permanent outage } \\
\text { rate (per year) }\end{array}$ \\
\hline 1 & 1 & 3 & 1.5 \\
2 & 2 & 4 & 5.0 \\
3 & 1 & 2 & 4.0 \\
4 & 3 & 4 & 1.0 \\
5 & 3 & 5 & 1.0 \\
6 & 1 & 3 & 1.5 \\
7 & 2 & 4 & 5.0 \\
8 & 4 & 5 & 1.0 \\
9 & 5 & 6 & 1.0 \\
\hline
\end{tabular}

Table 2. IEEE-RBTS(6 node) system generating set data.

\begin{tabular}{ccc}
\hline $\begin{array}{c}\text { Unit } \\
\text { Size(MW) }\end{array}$ & Type & $\begin{array}{c}\text { Fsilure Rate } \\
\text { Per year }\end{array}$ \\
\hline 5 & hydro & 2.0 \\
10 & thermal & 4.0 \\
20 & thermal & 5.0 \\
40 & thermal & 6.0 \\
\hline
\end{tabular}

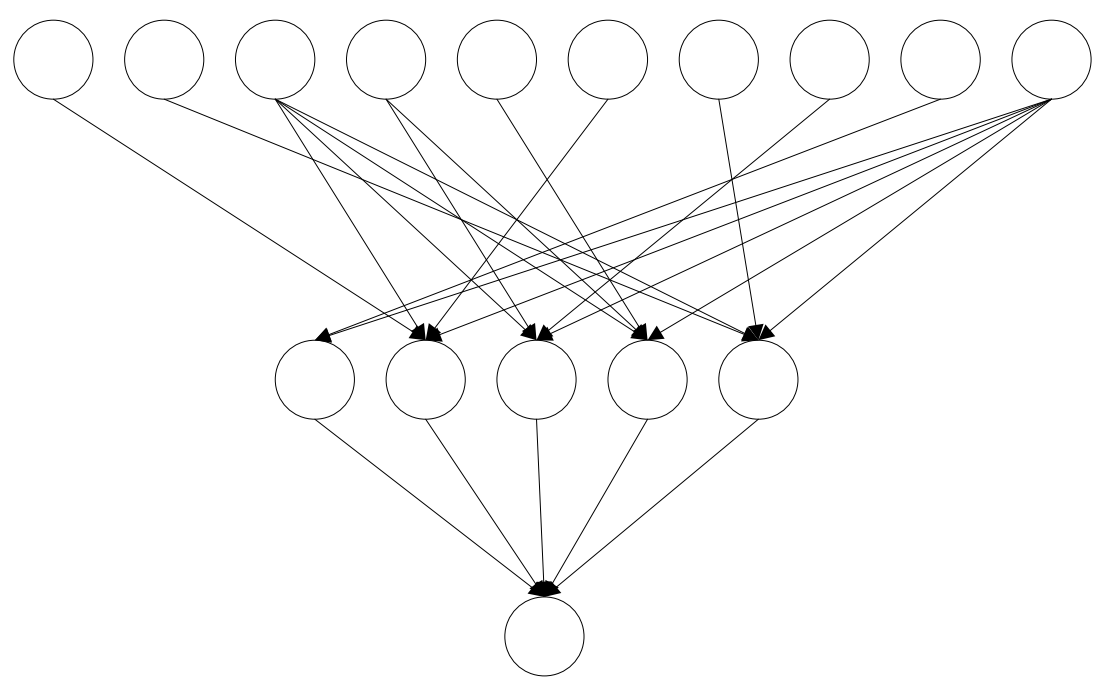

Figure 3. the minimal path of bayesian network(bus 2). 


\subsection{Bayesian Network Based on Minimal Cut Set Method}

Based on high-speed rail as shown in Figure 2 external power supply network, can respectively calculate the traction substation power supply of the minimum cut set, here in traction substation TPSS2 on bus 2 as an example, using Boolean determinant method, can get the minimum cut set of TPSS2: G1G2、 L1L3L6G2 、 L3L4L8G2 L3L4L5G2、L2L3L7G2. According to the minimum cut set of TPSS2 can make corresponding bayesian networks, shown as in Figure 4.

Based on bayesian networks is shown in Figure 4, combining with the development of the bayesian network reliability evaluation calculation program we can calculate $\mathrm{P}(\mathrm{T})_{2}$ :

$$
\mathrm{P}(\mathrm{T})_{2}=6.6348 \times 10^{-6}
$$

Among them, P (T) 2 is the outage probability of the traction substation on bus 2.Comparison shows that this result with bayesian network based on shortest path set method in the example calculation results $\mathrm{P}(\mathrm{T})_{2}$ is the same, in the same way,we can also verify TPSS3 TPSS6 power outage probability and minimum path set method of the calculation results are the same, therefore, by comparing the calculation results of the two methods, verify the feasibility of high speed railway external power supply reliability assessment and accurate.

\subsection{Bayesian network Identification System Vulnerabilities}

Because the bayesian network can bidirectional reasoning, so it can calculate when system failure, the posterior probability of each component, then through analysis the posteriori probability of each element, you can easily identify weak links of the system.

For high-speed rail external power supply system as shown in Figure 2, the posteriori probability of various components as shown in Table 3.

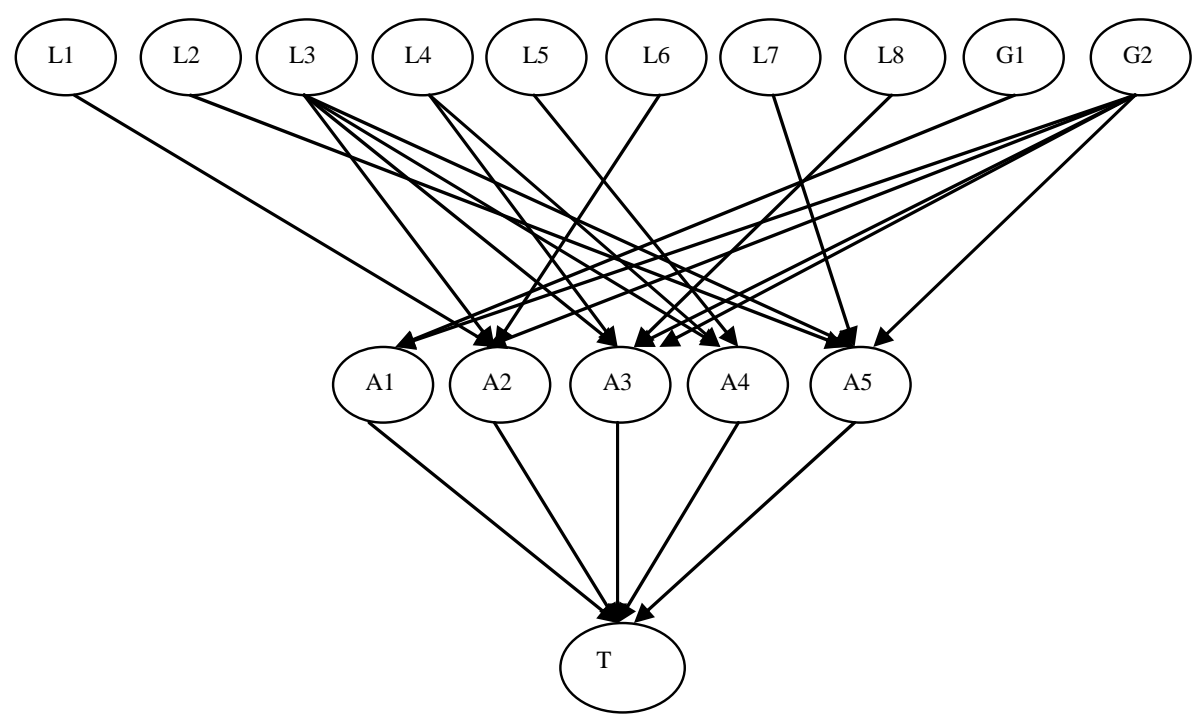

Figure 4. The minimal cut of bayesian network(bus 2).

Table 3. Main parts of posterior probability.

\begin{tabular}{cccccc}
\hline & Bus 2 & Bus 3 & Bus 4 & Bus 5 & Bus 6 \\
\hline L1 & $5.10 \times 10^{-4}$ & $4.30 \times 10^{-4}$ & $4.30 \times 10^{-4}$ & $4.30 \times 10^{-4}$ & $4.30 \times 10^{-4}$ \\
L2 & $1.43 \times 10^{-3}$ & $1.43 \times 10^{-3}$ & $1.43 \times 10^{-3}$ & $1.52 \times 10^{-3}$ & $1.43 \times 10^{-3}$ \\
L3 & $1.14 \times 10^{-3}$ & $1.14 \times 10^{-3}$ & $1.14 \times 10^{-3}$ & $1.13 \times 10^{-3}$ & $1.11 \times 10^{-3}$ \\
L4 & $2.90 \times 10^{-4}$ & $2.90 \times 10^{-4}$ & $2.90 \times 10^{-4}$ & $5 . .46 \times 10^{-4}$ & $2.96 \times 10^{-4}$ \\
L5 & $2.90 \times 10^{-4}$ & $2.90 \times 10^{-4}$ & $2.90 \times 10^{-4}$ & $1.28 \times 10^{-2}$ & $5.73 \times 10^{-4}$ \\
L6 & $4.3 \times 10^{-4}$ & $4.30 \times 10^{-4}$ & $4.30 \times 10^{-4}$ & $4.30 \times 10^{-4}$ & $4.30 \times 10^{-4}$ \\
L7 & $2.90 \times 10^{-4}$ & $1.43 \times 10^{-3}$ & $1.43 \times 10^{-3}$ & $1.52 \times 10^{-3}$ & $1.43 \times 10^{-3}$ \\
L8 & $2.90 \times 10^{-4}$ & $2.90 \times 10^{-4}$ & $2.90 \times 10^{-4}$ & $1.28 \times 10^{-2}$ & $5.73 \times 10^{-4}$ \\
G1 & 1 & 1 & 1 & 0.98725 & 0.97735 \\
G2 & 1 & 1 & 1 & 0.98744 & 0.02425 \\
L9 & 0 & 0 & 0 & 0 & 0.0257 \\
\hline
\end{tabular}


Through analysis posteriori probability of the various components in Table 3, high-speed railway external power of the weak link are L9 and G1, G2, so reduce the failure rate and maintenance time of generator $\mathrm{G} 1, \mathrm{G} 2$, and L9, will significantly improve the reliability of high speed railway external power supply.

\section{Conclusions}

1) Based on probabilistic inference of bayesian network used in high-speed railway external power supply reliability evaluation, established the minimum cut set and minimum path set method of bayesian network model, and develops the corresponding program for calculating the credible degree.

2) Using the IEEE RBTS system as an example to estimate the reliability of high speed railway external power supply scheme, and quantitative calculate the high-speed railway traction power substation of probability, proving the correctness and feasibility of the method which can be applied to high speed railway external power supply reliability evaluation.

3) Based on bidirectional reasoning technology of bayesian network, we can quantitative calculation the posterior probability of each elements in the external power supply system, and realize the identification of weakness about the external power supply, providing the basis for carrying out external power supply reliability distribution in the further.

\section{REFERENCES}

[1] M. W. Chen, "The Reliability Assessment of Traction Substation of High Speed Railway by the GO Methodol- ogy,” Power System Protection and Control, Vol. 39, No. 18, 2011, pp. 56-61.

[2] Y. Zhang, Z. Y. He and S. Lin, "A Power System Fault Diagnosis Method Based on DS Evidence Theory. Power System Protection and Control,” Vol. 36, No. 9, 2008, pp. 5-10.

[3] Y. F. Xie, "Reliability Assessment of Distribution Systems Based on Bayesian Networks," Baoding: Hebei Agricultural University, 2008.

[4] H. F. Su, "The Bayesian Networks and It's Application for Generation Systems Reliability Assessment,” Baoding : Hebei Agricultural University, 2004.

[5] X. W. Yin, W. X. Qian and L. Y. Xie, “A Method for System Reliability Assessment Based on Bayesian Networks," Aviation Journal, Vol. 29, No. 6, 2008, pp. 1482-1489.

[6] Q. Z. Luo, S. Shi, Y. H. Zhang and W. J. Liu, "The Application of Bayesian Network in Reliability Assessment of Multi-state System," Micro Computer Information, Vol. 26, No. 8-1, 2010, pp. 209-212.

[7] L. M. Huo, Y. L. Zhu, G. F. Fan, et al., “A Method Based on Bayesian Network Power System Reliability Assessment of the New Method," Automation of Electric Power Systems, Vol. 27, No. 5, 2003, pp. 36-40.

[8] W. X. Yin, "Reliability Evaluation of Mechanical System Based on Bayesian Network," Shenyang: Northeastern University. 2007.

[9] B. Xie, M. Z. Zhang and Y. X. Yan, "Improve Faulty Tree Analysis by Bayesian Networks," Journal of Yanshan University, Vol. 28, No. 1, 2004, pp. 55-58.

[10] R. Billinton and S. Kumar, "A Reliability Test System For Educational Purposes,” IEEE Transactions On Power Systems, Vol. 4, No. 3, pp. 1238-1244. doi: $10.1109 / 59.32623$ 